\title{
Biophysical Analysis of Binding of WW Domains of the YAP2 Transcriptional Regulator to PPXY Motifs within WBP1 and WBP2 Adaptors
}

\author{
Caleb B. McDonald, ${ }^{\dagger}$ Samantha K. N. McIntosh, ${ }_{\dagger}^{\dagger}$ David C. Mikles, ${ }^{\dagger}$ Vikas Bhat, ${ }^{\dagger}$ Brian J. Deegan, ${ }^{\dagger}$ \\ Kenneth L. Seldeen, ${ }^{\dagger}$ Ali M. Saeed, ${ }^{\dagger}$ Laura Buffa, ${ }^{\dagger}$ Marius Sudol, ${ }^{\ddagger}$, Zafar Nawaz, $^{\dagger}$ and Amjad Farooq*, ${ }^{\dagger}$ \\ ${ }^{\dagger}$ Department of Biochemistry and Molecular Biology and USylvester Braman Family Breast Cancer Institute, Leonard Miller School \\ of Medicine, University of Miami, Miami, Florida 33136, United States \\ Geisinger Clinic, Weis Center for Research, Danville, Pennsylvania 17822, United States \\ ${ }^{\S}$ Department of Medicine, Mount Sinai School of Medicine, New York, New York 10029, United States
}

ABSTRACT: The YAP2 transcriptional regulator mediates a plethora of cellular functions, including the newly discovered Hippo tumor suppressor pathway, by virtue of its ability to recognize $\mathrm{WBP} 1$ and $\mathrm{WBP} 2$ signaling adaptors among a wide variety of other ligands. Herein, using isothermal titration calorimery and circular dichroism in combination with molecular modeling and molecular dynamics, we provide evidence that the WW1 and WW2 domains of YAP2 recognize various PPXY motifs within WBP1 and WBP2 in a highly promiscuous and subtle manner. Thus, although both WW domains strictly require the integrity of the consensus PPXY sequence, nonconsensus residues within and flanking this motif are not critical for highaffinity binding, implying that they most likely play a role in

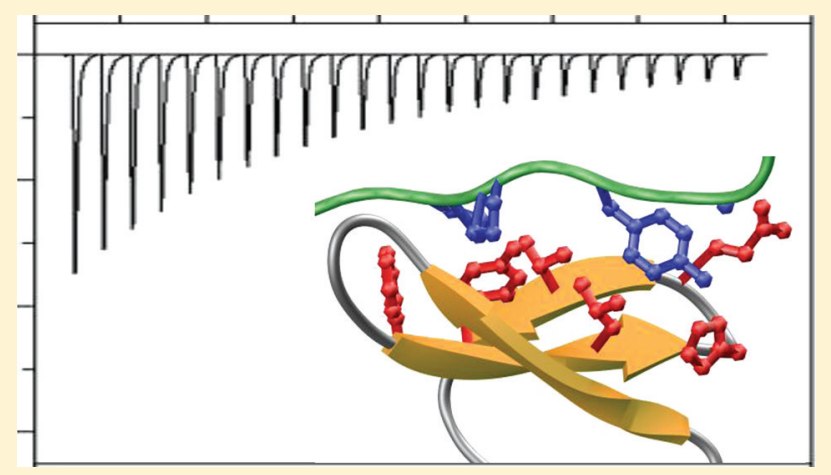
stabilizing the polyproline type II helical conformation of the PPXY ligands. Of particular interest is the observation that both WW domains bind to a PPXYXG motif with highest affinity, implicating a preference for a nonbulky and flexible glycine one residue to the C-terminal side of the consensus tyrosine. Importantly, a large set of residues within both WW domains and the PPXY motifs appear to undergo rapid fluctuations on a nanosecond time scale, suggesting that WW-ligand interactions are highly dynamic and that such conformational entropy may be an integral part of the reversible and temporal nature of cellular signaling cascades. Collectively, our study sheds light on the molecular determinants of a key WW-ligand interaction pertinent to cellular functions in health and disease.

YAP, originally identified as a binding partner of YES tyrosine kinase, ${ }^{1}$ is comprised of two major isoforms termed YAP1 and YAP2. While YAP2 contains a tandem copy of WW domains, termed WW1 and WW2, located N-terminal to the transactivation (TA) domain (Figure 1a), the WW2 domain is deleted in YAP1 through RNA splicing. ${ }^{2}$ YAP serves as a transcriptional regulator of a multitude of cellular factors, including p73, RUNX, TEAD, LATS1, and ErbB4, and, in particular, plays a key role in mediating the Hippo signaling pathway that is involved in regulating the size of organs and the suppression of tumors through inhibition of cellular proliferation and promotion of apoptosis. ${ }^{3-10}$ Consistent with these observations, YAP knockout in mice results in embryonic lethality. $^{11}$

Importantly, the WBP1 and WBP2 proline-rich proteins also rank among a wide diversity of YAP ligands. ${ }^{12,13}$ It has been previously demonstrated that the YAP-WBP interaction augments the transcriptional activity of the estrogen receptor and progesterone receptor in an E6AP-dependent manner. ${ }^{14}$ More recently, the YAP-WBP interaction has also been shown to play a key role in the Hippo tumor suppressor pathway. ${ }^{15-17}$ The WBP-YAP interaction is mediated by the canonical binding of WW domains of YAP to PPXY motifs located within the proline-rich (PR) domains of WBP proteins (Figure $1 b, c$ ). In fact, the WBP-YAP interaction was the first WW-ligand interaction characterized and led to identification of the PPXY consensus for class I WW domains. ${ }^{2,12,18-21}$ Remarkably, both WBP1 and WBP2 contain multiple copies of PPXY motifs, termed PY1 and PY2 and PY1-PY3, respectively. This raises the possibility that there may be multiple docking sites within WBP1 and WBP2 for accommodating YAP proteins, leading to the assembly of higher-order YAP-WBP multimers rather than simple binary complexes. Additionally, the fact that YAP2 contains a tandem copy of WW domains may also favor the formation of YAP2-WBP complexes through a bidentate mechanism, resulting in an affinity much higher than that

Received: August 12, 2011

Revised: October 3, 2011

Published: October 10, 2011 
a

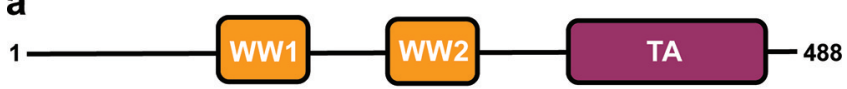

b
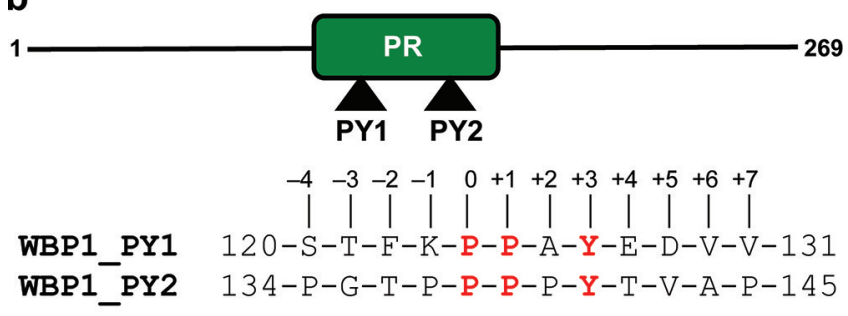

C

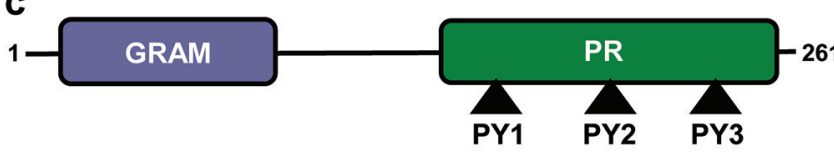

$\begin{array}{ll} & \\ \text { WBP2 PY1 } & 163-\mathrm{M}-\mathrm{Y}-\mathrm{P}-\mathrm{C}-\mathrm{P}-\mathrm{P}-\mathrm{G}-\mathrm{Y}-\mathrm{P}-\mathrm{Y}-\mathrm{P}-\mathrm{P}-174 \\ \text { WBP2 }-\mathrm{PY} 2 & 193-\mathrm{V}-\mathrm{Q}-\mathrm{P}-\mathrm{P}-\mathrm{P}-\mathrm{P}-\mathrm{P}-\mathrm{Y}-\mathrm{P}-\mathrm{G}-\mathrm{P}-\mathrm{M}-204 \\ \text { WBP2 } 2 \text { PY3 } & 245-\mathrm{S}-\mathrm{Q}-\mathrm{P}-\mathrm{P}-\mathrm{P}-\mathrm{P}-\mathrm{P}-\mathrm{Y}-\mathrm{Y}-\mathrm{P}-\mathrm{P}-\mathrm{E}-256\end{array}$

Figure 1. Modular organization of the human YAP2 transcriptional regulator and human WBP proteins. (a) YAP2 is comprised of a tandem copy of WW domains, designated WW1 and WW2, located Nterminal to the transactivation (TA) domain. (b) WBP1 contains a central proline-rich (PR) domain flanked by long stretches of uncharacterized regions. The PR domain of WBP1 contains two PPXY motifs, designated PY1 and PY2. (c) WBP2 contains the GRAM domain located $\mathrm{N}$-terminal to the proline-rich (PR) domain. The PR domain of WBP2 contains three PPXY motifs, designated PY1-PY3. Note that the amino acid sequences of peptides containing the PPXY motifs and flanking residues within both WBP1 and WBP2 are provided. The numerals indicate the nomenclature used in this study to distinguish residues within and flanking the motifs relative to the first proline within the PPXY motifs, which is arbitrarily assigned position 0 .

afforded by the binding of a single WW domain as in the case of YAP1. This argument is further supported by the observation that YAP2 is a more potent transcriptional activator than YAP1. ${ }^{4,22}$

In an effort to lay the groundwork for elucidating the molecular basis of the YAP-WBP interaction, we report here detailed thermodynamic and structural analysis of the binding of WW1 and WW2 domains of YAP2 to PPXY peptides derived from $\mathrm{WBP} 1$ and $\mathrm{WBP} 2$ using isothermal titration calorimery (ITC) and circular dichroism (CD) in combination with molecular modeling (MM) molecular dynamics (MD). Our data reveal that the WW1 and WW2 domains of YAP2 recognize various PPXY motifs within WBP1 and WBP2 in a highly promiscuous and subtle manner. Thus, although both WW domains strictly require the integrity of the consensus PPXY sequence, nonconsensus residues within and flanking this motif are not critical for high-affinity binding, implying that they most likely play a role in stabilizing the polyproline type II (PPII) helical conformation of the PPXY ligands. Of particular interest is the observation that both WW domains bind to a PPXYXG motif with highest affinity, implicating a preference for a nonbulky and flexible glycine one residue to the $\mathrm{C}$ terminal side of the consensus tyrosine. Importantly, a large set of residues within both WW domains and the PPXY motifs appear to undergo rapid fluctuations on a nanosecond time scale, suggesting that WW-ligand interactions are highly dynamic and that such conformational entropy may be an integral part of the reversible and temporal nature of cellular signaling cascades. Collectively, our study sheds light on the molecular determinants of a key WW-ligand interaction pertinent to cellular functions in health and disease.

\section{MATERIALS AND METHODS}

Protein Preparation. WW1 (residues 171-205) and WW2 (residues 230-264) domains of human YAP2 were cloned into pET30 bacterial expression vectors with an $\mathrm{N}$ terminal His tag using Novagen LIC technology. The proteins were subsequently expressed in Escherichia coli strain BL21*(DE3) (Invitrogen) and purified on a Ni-NTA affinity column using standard procedures. Briefly, bacterial cells were grown at $20^{\circ} \mathrm{C}$ in Terrific Broth to an optical density of $>1$ at $600 \mathrm{~nm}$ prior to induction with $0.5 \mathrm{mM}$ isopropyl $\beta$-D-1thiogalactopyranoside (IPTG). The bacterial culture was further grown overnight at $20{ }^{\circ} \mathrm{C}$, and the cells were subsequently harvested and disrupted using a BeadBeater (Biospec). After separation of cell debris via high-speed centrifugation, the cell lysate was loaded onto a Ni-NTA column and washed extensively with $20 \mathrm{mM}$ imidazole to remove nonspecific binding of bacterial proteins to the column. The recombinant proteins were subsequently eluted with 200 $\mathrm{mM}$ imidazole and dialyzed against an appropriate buffer to remove excess imidazole. Further treatment on a Hiload Superdex 200 size-exclusion chromatography (SEC) column coupled in line with a GE Akta fast performance liquid chromatography system led to purification of WW domains to apparent homogeneity as judged by sodium dodecyl sulfatepolyacrylamide gel electrophoresis analysis. The final yield was typically between 50 and $100 \mathrm{mg}$ of protein of apparent homogeneity per liter of bacterial culture. Protein concentrations were determined by the fluorescence-based Quant-It assay (Invitrogen) and spectrophotometrically using extinction coefficients of 12490 and $13980 \mathrm{M}^{-1} \mathrm{~cm}^{-1}$ calculated for the WW1 and WW2 domains, respectively, of YAP2 using the online software ProtParam at ExPasy Server. ${ }^{23}$ Results from both methods were in an excellent agreement.

Peptide Synthesis. The 12-mer wild-type and mutant peptides spanning various PPXY motifs within human WBP1 and WBP2 proteins were commercially obtained from GenScript Corp. The sequences of these peptides are shown in panels $b$ and $c$ of Figure 1. The peptide concentrations were measured gravimetrically.

Isothermal Titration Calorimetry. Isothermal titration calorimetry (ITC) experiments were performed on a Microcal VP-ITC instrument, and data were acquired and processed using fully automated features in Microcal Origin. All measurements were repeated at least three times. Briefly, WW domain samples were prepared in $50 \mathrm{mM}$ sodium phosphate, $100 \mathrm{mM} \mathrm{NaCl}, 1 \mathrm{mM}$ EDTA, and $5 \mathrm{mM} \beta$ mercaptoethanol ( $\mathrm{pH}$ 7.0). The experiments were initiated by injection of $25 \times 10 \mu \mathrm{L}$ aliquots of each peptide at $2-4 \mathrm{mM}$ from the syringe into the calorimetric cell containing $1.8 \mathrm{~mL}$ of a $100-200 \mu \mathrm{M} W \mathrm{~W}$ domain solution at $25^{\circ} \mathrm{C}$. The change in thermal power as a function of each injection was automatically recorded using Origin, and the raw data were further processed to yield binding isotherms of heat release per injection as a function of molar ratio of each peptide to the WW domain. The 

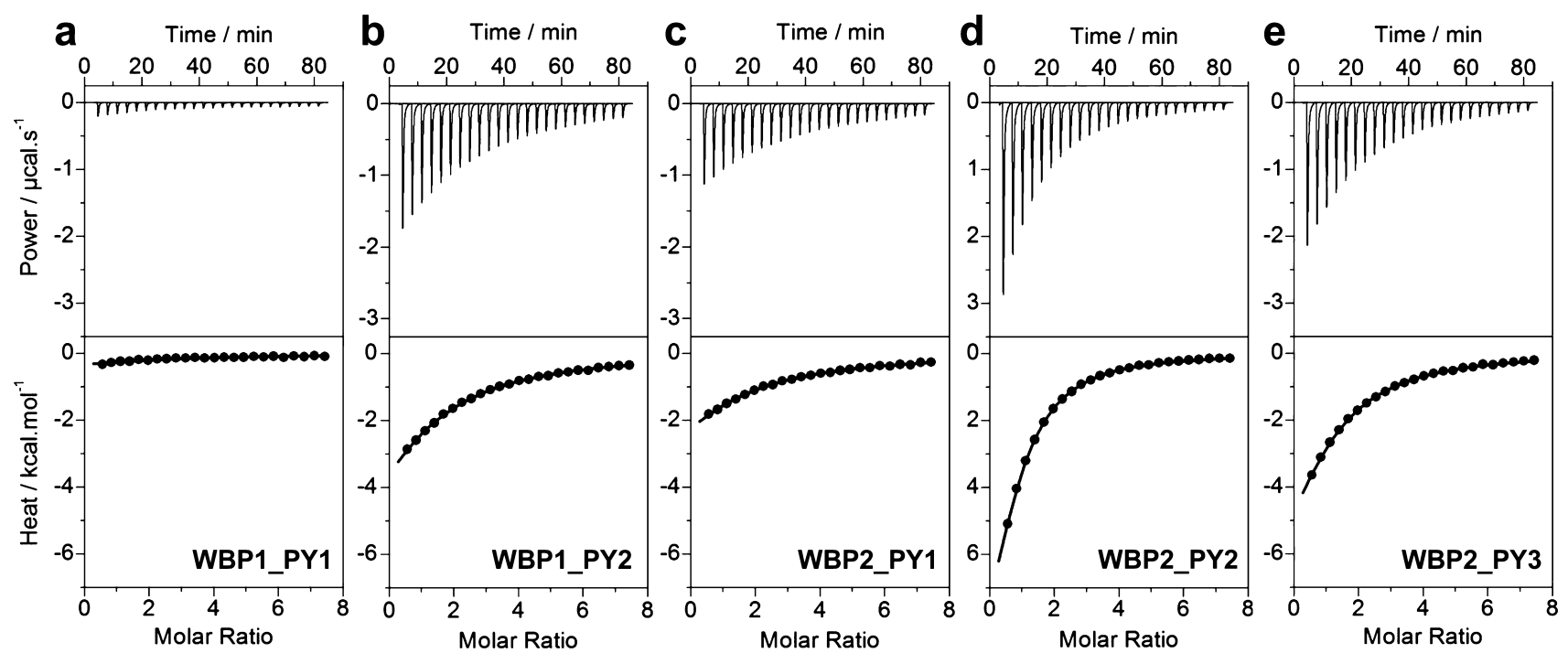

Figure 2. Representative ITC isotherms for the binding of the WW1 domain of YAP2 to PPXY peptides containing WBP1_PY1 (a), WBP1_PY2 (b), WBP2_PY1 (c), WBP2_PY2 (d), and WBP2_PY3 (e) motifs. The top panels show the raw ITC data expressed as the change in thermal power with respect to time over the period of titration. In the bottom panels, the change in molar heat is expressed as a function of the molar ratio of the corresponding peptide to the WW1 domain of YAP2. The solid lines in the bottom panels show the fit of data to a one-site model, as embodied in eq 1 , using Origin.

heats of mixing and dilution were subtracted from the heat of binding per injection by conducting a control experiment in which the same buffer in the calorimetric cell was titrated against each peptide in an identical manner. To extract binding affinity $\left(K_{\mathrm{d}}\right)$ and binding enthalpy $(\Delta H)$, the ITC isotherms were iteratively fit to the following built-in function by nonlinear least-squares regression analysis using integrated Origin:

$$
\begin{aligned}
q(i)= & (n \Delta H V P / 2)\left\{1+L / n P+K_{\mathrm{d}} / n P\right. \\
& \left.-\left[\left(1+L / n P+K_{\mathrm{d}} / n P\right)^{2}-4 L / n P\right]^{1 / 2}\right\}
\end{aligned}
$$

where $q(i)$ is the heat release (kilocalories per mole) for the $i$ th injection, $n$ is the binding stoichiometry, $V$ is the effective volume of the protein solution in the calorimetric cell (1.46 $\mathrm{mL}), P$ is the total protein concentration in the calorimetric cell, and $L$ is the total concentration of peptide ligand added for the $i$ th injection. Equation 1 is derived from the binding of a ligand to a macromolecule using the law of mass action assuming a one-site model. ${ }^{24}$ The free energy change $(\Delta G)$ upon ligand binding was calculated from the relationship

$$
\Delta G=R T \ln K_{\mathrm{d}}
$$

where $R$ is the universal molar gas constant $\left(1.99 \mathrm{cal} \mathrm{K}^{-1}\right.$ $\mathrm{mol}^{-1}$ ) and $T$ is the absolute temperature. The entropic contribution $(T \Delta S)$ to the free energy of binding was calculated from the relationship

$$
T \Delta S=\Delta H-\Delta G
$$

where $\Delta H$ and $\Delta G$ are as defined above.

Circular Dichroism. Circular dichroism (CD) measurements were taken on a Bio-Logic MOS450 spectropolarimeter thermostatically controlled with a water bath at $25{ }^{\circ} \mathrm{C}$. Experiments were conducted on a $100 \mu \mathrm{M}$ peptide solution in $10 \mathrm{mM}$ sodium phosphate $(\mathrm{pH} 7.0)$. Data were collected using a quartz cuvette with a $2 \mathrm{~mm}$ path length in the wavelength range of $180-260 \mathrm{~nm}$ and processed using integrated BIOKINE. Data were normalized against reference spectra to remove the contribution of buffer. The reference spectra were obtained in a similar manner with $10 \mathrm{mM}$ sodium phosphate ( $\mathrm{pH} 7.0$ ). Data were recorded with a slit bandwidth of $2 \mathrm{~nm}$ at a scan rate of $3 \mathrm{~nm} / \mathrm{min}$. Each data set represents an average of four scans acquired at $1 \mathrm{~nm}$ intervals. Data were converted to molar ellipticity, $[\theta]$, as a function of wavelength $(\lambda)$ of electromagnetic radiation using the equation

$$
[\theta]=\left(10^{5} \Delta \varepsilon\right) / c l \operatorname{deg~} \mathrm{cm}^{2} \mathrm{dmol}^{-1}
$$

where $\Delta \varepsilon$ is the observed ellipticity in millidegrees, $c$ is the peptide or protein concentration in micromolar, and $l$ is the cuvette path length in centimeters.

Macromolecular Modeling. Macromolecular modeling (MM) was employed to build three-dimensional (3D) structures of WW1 and WW2 domains of YAP2 in complex with a peptide containing the PY2 motif of WBP2 (WBP2_PY2) using MODELER based on homology modeling. $^{25}$ In each case, the NMR structure of the WW domain of YAP1 bound to a peptide containing the PPXY motif was used as a template (Protein Data Bank entry 1JMQ). A total of 100 atomic models were calculated, and the structure with the lowest energy, as judged by the MODELER Objective Function, was selected for further analysis. The atomic models were rendered using RIBBONS. ${ }^{26}$ It is important to note that the WW domain of YAP1 shares $100 \%$ amino acid sequence identity with the WW1 domain of YAP2, while it is close to $50 \%$ identical to the WW2 domain of YAP2. Given such high levels of sequence identity between the target and template WW domains coupled with virtually identical target and template peptide ligands sharing the PPXY consensus motif, the $3 \mathrm{D}$ structural models presented here can be used with a high degree of confidence at atomic resolution.

Molecular Dynamics. Molecular dynamics (MD) simulations were performed with GROMACS ${ }^{27,28}$ using the integrated OPLS-AA force field. ${ }^{29,30}$ Briefly, the modeled structures of the WW1 domain of YAP2 in complex with various PPXY peptides derived from WBP1 and WBP2 adaptors were centered within a cubic box and hydrated 


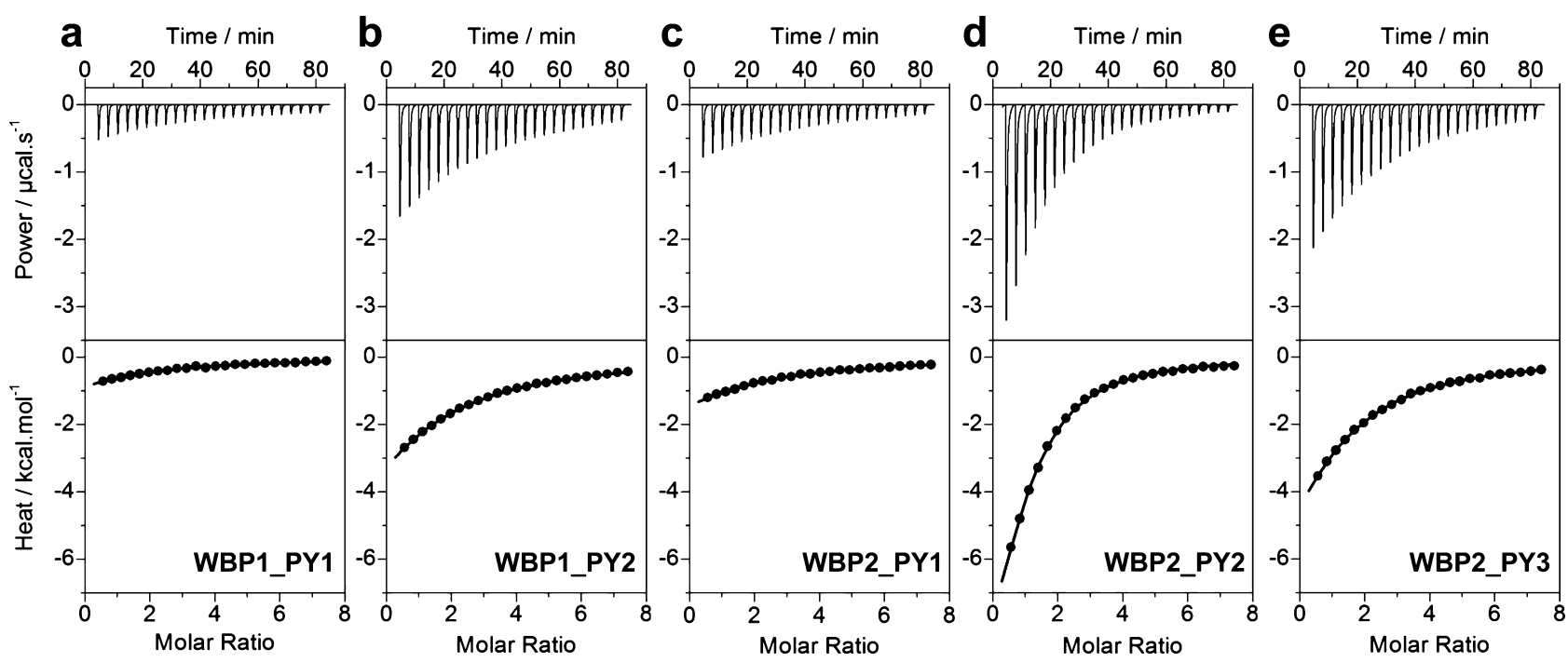

Figure 3. Representative ITC isotherms for the binding of the WW2 domain of YAP2 to PPXY peptides containing WBP1 PY1 (a), WBP1 PY2 (b), WBP2_PY1 (c), WBP2_PY2 (d), and WBP2_PY3 (e) motifs. The top panels show the raw ITC data expressed as the change in thermal power with respect to time over the period of titration. In the bottom panels, the change in molar heat is expressed as a function of the molar ratio of corresponding peptide to the WW2 domain of YAP2. The solid lines in the bottom panels show the fit of data to a one-site model, as embodied in eq 1 , using Origin.

Table 1. Thermodynamic Parameters Obtained from ITC Measurements for the Binding of the WW1 Domain of YAP2 to PPXY Peptides Derived from WBP1 and WBP2 ${ }^{a}$

$\begin{array}{clrrrr}\text { peptide } & \text { sequence } & K_{\mathrm{d}}(\mu \mathrm{M}) & \Delta H(\mathrm{kcal} / \mathrm{mol}) & T \Delta S(\mathrm{kcal} / \mathrm{mol}) & \Delta G(\mathrm{kcal} / \mathrm{mol}) \\ \text { WBP1_PY1 } & \text { STFKPPAYEDVV } & 320 \pm 6 & -2.39 \pm 0.07 & 2.38 \pm 0.08 & -4.77 \pm 0.01 \\ \text { WBP1_PY2 } & \text { PGTPPPPYTVAP } & 132 \pm 1 & -11.59 \pm 0.23 & -6.29 \pm 0.24 & -5.30 \pm 0.01 \\ \text { WBP2_PY1 } & \text { MYPCPPGYPYPP } & 168 \pm 6 & -9.20 \pm 0.11 & -4.05 \pm 0.13 & -5.15 \pm 0.02 \\ \text { WBP2_PY2 } & \text { VQPPPPPYPGPM } & 40 \pm 1 & -13.35 \pm 0.11 & -7.33 \pm 0.12 & -6.01 \pm 0.01 \\ \text { WBP2_PY3 } & \text { SQPPPPPYYPPE } & 74 \pm 1 & -10.67 \pm 0.16 & -5.03 \pm 0.16 & -5.64 \pm 0.01\end{array}$

${ }^{a}$ Binding stoichiometries generally agreed to within $\pm 10 \%$. Errors were calculated from at least three independent measurements. All errors are given to one standard deviation.

Table 2. Thermodynamic Parameters Obtained from ITC Measurements for the Binding of the WW2 Domain of YAP2 to PPXY Peptides Derived from WBP1 and WBP2 ${ }^{a}$

$\begin{array}{clrrrr}\text { peptide } & \text { sequence } & K_{\mathrm{d}}(\mu \mathrm{M}) & \Delta H(\mathrm{kcal} / \mathrm{mol}) & T \Delta S(\mathrm{kcal} / \mathrm{mol}) & \Delta G(\mathrm{kcal} / \mathrm{mol}) \\ \text { WBP1_PY1 } & \text { STFKPPAYEDVV } & 194 \pm 6 & -4.31 \pm 0.39 & 0.77 \pm 0.41 & -5.07 \pm 0.02 \\ \text { WBP1_PY2 } & \text { PGTPPPPYTVAP } & 193 \pm 8 & -14.13 \pm 0.06 & -9.06 \pm 0.03 & -5.07 \pm 0.03 \\ \text { WBP2_PY1 } & \text { MYPCPPGYPYPP } & 231 \pm 4 & -7.80 \pm 0.28 & -2.83 \pm 0.26 & -4.97 \pm 0.01 \\ \text { WBP2_PY2 } & \text { VQPPPPPYPGPM } & 43 \pm 1 & -13.18 \pm 0.09 & -7.21 \pm 0.09 & -5.96 \pm 0.01 \\ \text { WBP2_PY3 } & \text { SQPPPPPYYPPE } & 116 \pm 1 & -13.51 \pm 0.23 & -8.14 \pm 0.22 & -5.37 \pm 0.01\end{array}$

${ }^{a}$ Binding stoichiometries generally agreed to within $\pm 10 \%$. Errors were calculated from at least three independent measurements. All errors are given to one standard deviation.

using the extended simple point charge (SPC/E) water model. ${ }^{31,32}$ The hydrated structures were energy-minimized with the steepest descent algorithm prior to equilibration under the NPT ensemble conditions, wherein the number of atoms $(N)$, pressure $(P)$, and temperature $(T)$ within the system were kept constant at $\sim 17000,1 \mathrm{bar}$, and $300 \mathrm{~K}$, respectively. The particle-mesh Ewald (PME) method was employed to compute long-range electrostatic interactions with a $10 \AA$ cutoff $^{33}$ and the Linear Constraint Solver (LINCS) algorithm to restrain bond lengths. ${ }^{34}$ All MD simulations were performed under periodic boundary conditions (PBC) using the leapfrog integrator with a time step of $2 \mathrm{fs}$. For the final MD production runs, data were collected every $10 \mathrm{ps}$ over a time scale of 100 ns.

\section{RESULTS AND DISCUSSION}

WW Domains of YAP2 Bind to PPXY Motifs within WBP1 and WBP2 with Differential Affinities. To understand YAP2-WBP interaction in quantitative terms, we analyzed the binding of WW domains of YAP2 to PPXY peptides derived from potential YAP2 binding sites in WBP1 and WBP2 using ITC (Figures 2 and 3). Detailed thermodynamic parameters obtained from such measurements are provided in Tables 1 and 2. Our analysis reveals that while both WW domains of YAP2 bind in a physiologically relevant manner to all PPXY motifs within both WBP1 and WBP2, they do so with differential affinities. Importantly, both WW domains recognize the PY2 and PY3 motifs within WBP2 with much higher affinities than the PY1 motif in WBP2 and 
Table 3. Thermodynamic Parameters Obtained from ITC Measurements for the Binding of the WW1 Domain of YAP2 to the Wild Type (WT) and Single-Alanine Mutants of the WBP2_PY3 Peptide ${ }^{a}$

\begin{tabular}{llcrrr}
\multicolumn{1}{c}{ peptide } & sequence & $K_{\mathrm{d}}(\mu \mathrm{M})$ & $\Delta H(\mathrm{kcal} / \mathrm{mol})$ & $T \Delta S(\mathrm{kcal} / \mathrm{mol})$ & $\Delta G(\mathrm{kcal} / \mathrm{mol})$ \\
PY3_WT & SQPPPPPYYPPE & $74 \pm 1$ & $-10.67 \pm 0.16$ & $-5.03 \pm 0.16$ & $-5.64 \pm 0.01$ \\
PY3_Q-3A & SAPPPPPYYPPE & $75 \pm 1$ & $-8.03 \pm 0.09$ & $-2.39 \pm 0.09$ & $-5.64 \pm 0.01$ \\
PY3_P-2A & SQAPPPPYYPPE & $117 \pm 8$ & $-12.97 \pm 0.34$ & $-7.60 \pm 0.30$ & $-5.37 \pm 0.04$ \\
PY3_P-1A & SQPAPPPYYPPE & $88 \pm 1$ & $-9.06 \pm 0.01$ & $-3.52 \pm 0.01$ & $-5.54 \pm 0.01$ \\
PY3_P0A & SQPPAPPYYPPE & $328 \pm 25$ & $-8.32 \pm 0.11$ & $-3.56 \pm 0.15$ & $-4.76 \pm 0.05$ \\
PY3_P1A & SQPPPA_PYYPPE & $409 \pm 19$ & $-2.75 \pm 0.29$ & $1.88 \pm 0.32$ & $-4.63 \pm 0.03$ \\
PY3_P2A & SQPPPP_AYYPPE & $107 \pm 4$ & $-6.31 \pm 0.04$ & $-0.88 \pm 0.06$ & $-5.42 \pm 0.02$ \\
PY3_Y3A & SQPPPPP_AYPPE & $201 \pm 1$ & $-3.32 \pm 0.05$ & $-73 \pm 0.05$ & $-5.05 \pm 0.01$ \\
PY3_Y4A & SQPPPPPYAPPE & $73 \pm 3$ & $-10.34 \pm 0.04$ & $-4.69 \pm 0.07$ & $-5.65 \pm 0.02$ \\
PY3_P5A & SQPPPPPYYAPE & $57 \pm 1$ & $-10.66 \pm 0.23$ & $-4.86 \pm 0.25$ & $-5.80 \pm 0.01$ \\
PY3_P6A & SQPPPPPYYPAE & $76 \pm 1$ & $-10.94 \pm 0.12$ & $-5.31 \pm 0.13$ & $-5.62 \pm 0.01$
\end{tabular}

${ }^{a_{T}}$ The alanine substitutions within the WBP2_PY3 peptide are underlined for the sake of clarity. Binding stoichiometries generally agreed to within $\pm 10 \%$. Errors were calculated from at least three independent measurements. All errors are given to one standard deviation.

Table 4. Thermodynamic Parameters Obtained from ITC Measurements for the Binding of the WW2 Domain of YAP2 to the Wild Type (WT) and Single-Alanine Mutants of the WBP2_PY3 Peptide ${ }^{a}$

\begin{tabular}{llcrrr}
\multicolumn{1}{c}{ peptide } & sequence & $K_{\mathrm{d}}(\mu \mathrm{M})$ & $\Delta H(\mathrm{kcal} / \mathrm{mol})$ & $T \Delta S(\mathrm{kcal} / \mathrm{mol})$ & $\Delta G(\mathrm{kcal} / \mathrm{mol})$ \\
PY3_WT & SQPPPPPYYPPE & $116 \pm 1$ & $-13.51 \pm 0.23$ & $-8.14 \pm 0.22$ & $-5.37 \pm 0.01$ \\
PY3_Q-3A & SAPPPPPYYPPE & $107 \pm 4$ & $-8.92 \pm 0.01$ & $-3.49 \pm 0.03$ & $-5.42 \pm 0.02$ \\
PY3_P-2A & SQAPPPPPYPPE & $111 \pm 3$ & $-13.53 \pm 0.03$ & $-8.13 \pm 0.01$ & $-5.40 \pm 0.02$ \\
PY3_P-1A & SQPAPPPYYPPE & $117 \pm 1$ & $-8.97 \pm 0.08$ & $-3.60 \pm 0.08$ & $-5.37 \pm 0.01$ \\
PY3_P0A & SQPPAPPYYPPE & $368 \pm 18$ & $-9.60 \pm 0.20$ & $-4.91 \pm 0.17$ & $-4.69 \pm 0.03$ \\
PY3_P1A & SQPPP_APYYPPE & $590 \pm 44$ & $-4.61 \pm 0.28$ & $-0.19 \pm 0.23$ & $-4.41 \pm 0.04$ \\
PY3_P2A & SQPPPP_AYYPPE & $163 \pm 9$ & $-8.02 \pm 0.06$ & $-2.84 \pm 0.10$ & $-5.17 \pm 0.03$ \\
PY3_Y3A & SQPPPPPAYPPE & $199 \pm 8$ & $-3.46 \pm 0.27$ & $1.60 \pm 0.25$ & $-5.06 \pm 0.02$ \\
PY3_Y4A & SQPPPPPYAPPE & $104 \pm 2$ & $-9.85 \pm 0.18$ & $-4.41 \pm 0.17$ & $-5.44 \pm 0.01$ \\
PY3_P5A & SQPPPPPYYAPE & $79 \pm 4$ & $-10.43 \pm 0.35$ & $-4.82 \pm 0.38$ & $-5.61 \pm 0.03$ \\
PY3_P6A & SQPPPPPYYPAE & $105 \pm 2$ & $-12.41 \pm 0.03$ & $-6.97 \pm 0.04$ & $-5.44 \pm 0.01$
\end{tabular}

${ }^{a_{\text {The }}}$ alanine substitutions within the WBP2_PY3 peptide are underlined for the sake of clarity. Binding stoichiometries generally agreed to within $\pm 10 \%$. Errors were calculated from at least three independent measurements. All errors are given to one standard deviation.

PY1 and PY2 motifs in WBP1. Despite these differences, binding of both WW domains of YAP2 to all PPXY motifs is predominantly driven by favorable enthalpic forces accompanied by unfavorable entropic changes, except for their interaction with the PY1 motif of WBP1, wherein entropic factors also contribute favorably. Notably, the overall enthalpically driven nature of the WW-PPXY interaction suggests the formation of specific intermolecular interactions such as hydrogen bonding and van der Waals contacts that likely account for the specificity of this key protein-protein interaction. In contrast, the overall unfavorable entropic changes most likely result from the loss of degrees of freedom available to both partners upon intermolecular association.

In an attempt to fully understand the energetic contribution of residues within and flanking the PPXY motifs derived from WBP1 and WBP2 toward the binding of WW domains of YAP2, we next performed an alanine scan on the peptide containing the PY3 motif of WBP2 (WBP2_PY3) and measured the binding of each alanine mutant to both WW domains of YAP2 (Tables 3 and 4). Surprisingly, our data show that alanine substitution of nonconsensus residues within and flanking the PPXY motif has little or negligible effect on the binding affinity of both WW domains. This salient observation implies that nonconsensus residues within and flanking the PPXY motifs are not critically required for driving the YAP2WBP interaction, but they may be important for stabilizing the conformation of PPXY peptides. Interestingly, the P5A alanine substitution, according to the nomenclature presented in panels $\mathrm{b}$ and $\mathrm{c}$ of Figure 1, within WBP2_PY3 enhances binding to both WW domains, suggesting that an amino acid with a small nonbulky side chain is preferred at this position. This notion is further corroborated by the observation that the PY2 motif of WBP2 (WBP2_PY2) not only contains a nonbulky glycine at position 5 but also binds to both WW domains with the highest affinity among all PPXY peptides examined. These observations are in agreement with a previous study in which substitution of specific residues flanking the PPXY motif enhanced the binding of WBP1_PY2 to the WW domain of YAP1 by 1 order of magnitude, ${ }^{35}$ implying that residues within and flanking the PPXY motifs are likely to both enhance and destabilize the WBP-YAP interaction.

Taken together, our thermodynamic data suggest strongly that although nonconsensus residues within and flanking the PPXY motifs are not critical for high-affinity binding to WW domains, some residues at these positions may be more destabilizing than others either through their engagement in unfavorable intermolecular contacts or by simply compromising the peptide conformation that best fits the WW domains. Thus, for example, our data suggest that the residue at position 5 with a bulky side chain within the PPXY motifs may be destabilizing. Indeed, all PPXY motifs but the WBP2_PY2 motif contain bulky residues at position 5 , and their binding to WW domains is compromised relative to the binding of the WBP2 PY2 motif. In a similar manner, the PY1 motif of $\overline{\mathrm{WBP}} 2$ 

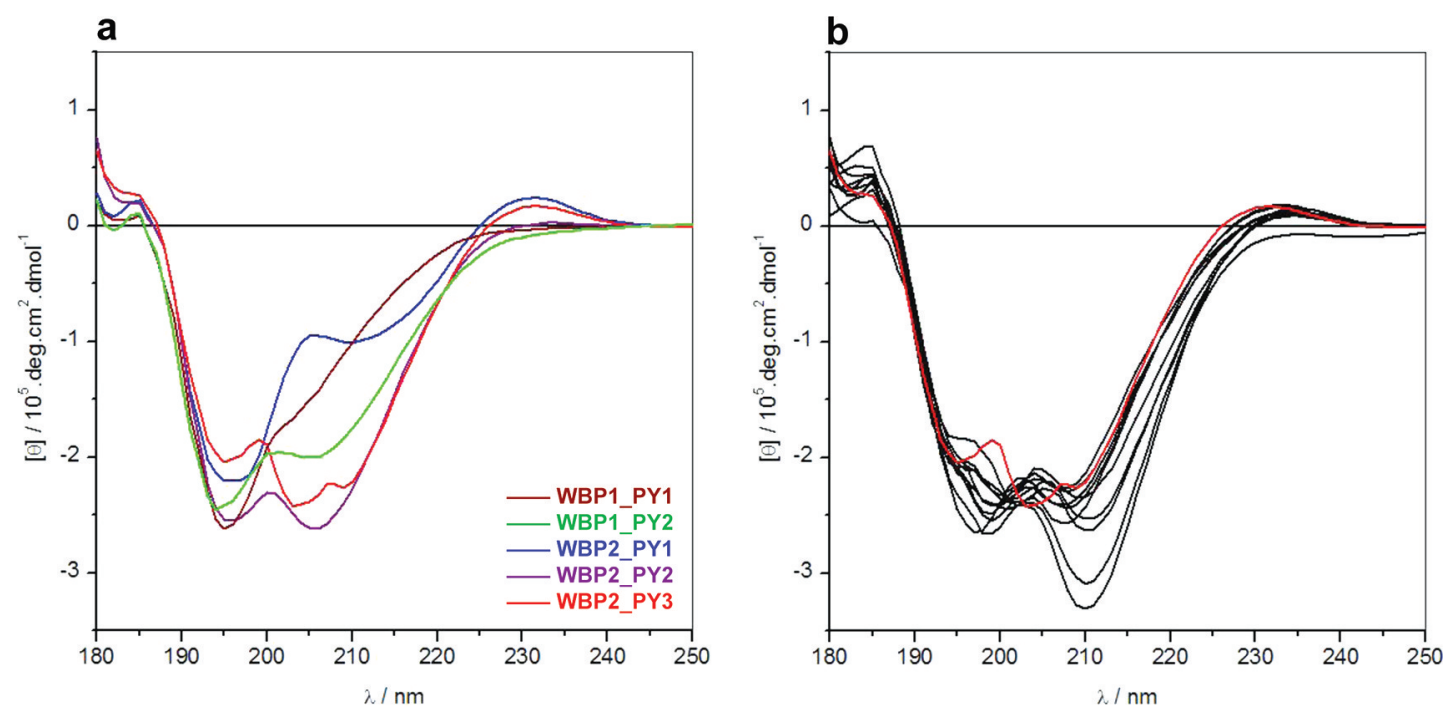

Figure 4. CD analysis of PPXY peptides derived from WBP1 and WBP2 proteins. (a) Comparison of far-UV CD spectra of WBP1_PY1 (brown), WBP1_PY2 (green), WBP2_PY1 (blue), WBP2_PY2 (purple), and WBP2_PY3 (red). (b) Comparison of far-UV CD spectra of the wild-type WBP2_PY3 peptide (red) and single-alanine mutant peptides thereof (black).
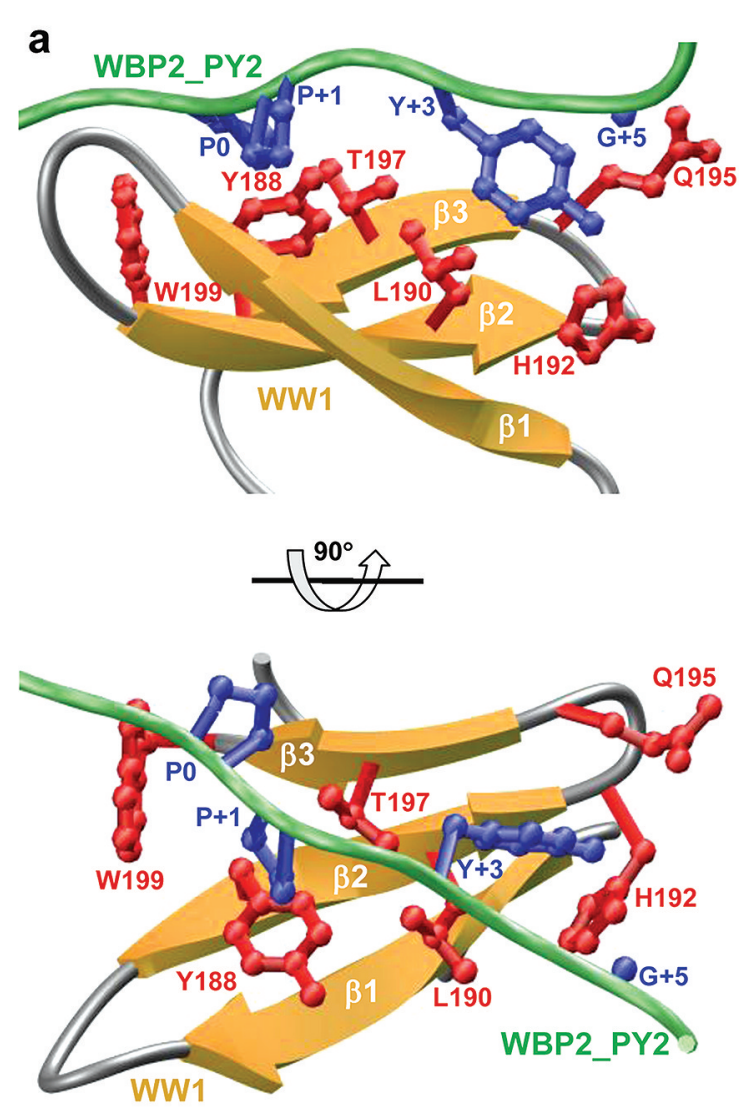

b
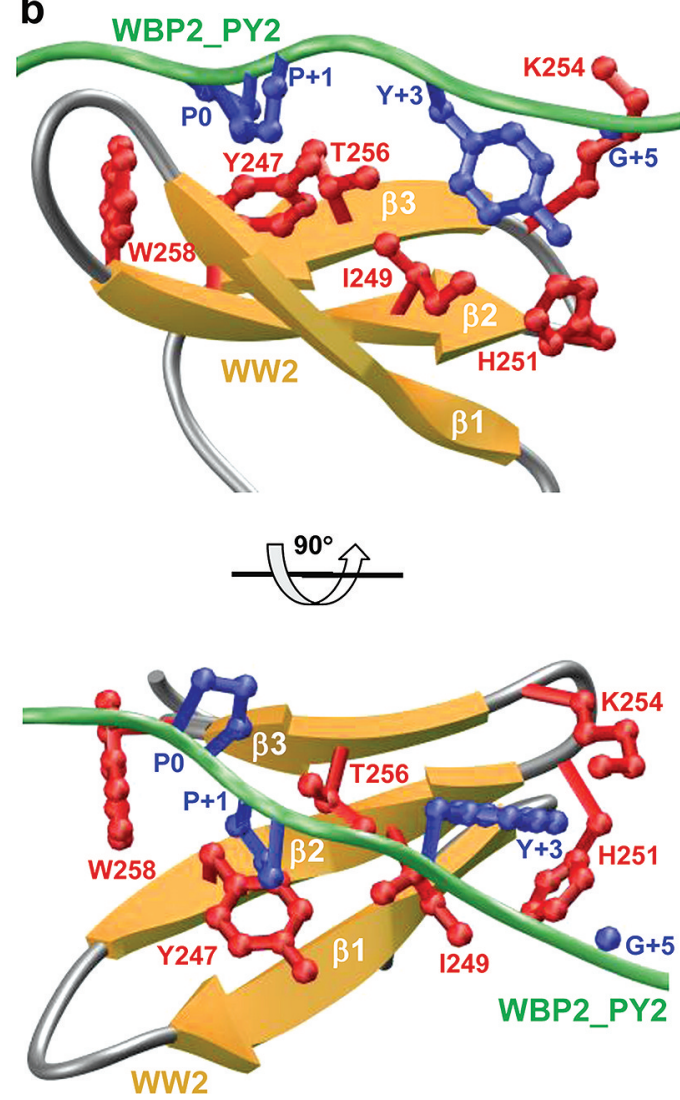

Figure 5. 3D structural models of the WW1 (a) and WW2 (b) domains of YAP2 in complex with the WBP2_PY2 peptide. The $\beta$-strands in the WW domains are colored yellow with loops colored gray and the peptide colored green. The side chain moieties of residues within WW domains engaged in key intermolecular contacts with the peptide are colored red. The side chain moieties of residues within the peptide colored blue correspond to the PPXYXG motif.

(WBP2_PY1) and the PY1 and PY2 motifs of WBP1 (WBP1_PY1 and WBP1_PY2, respectively) bind to WW domains with much weaker affinities relative to those of WBP2_PY2 and WBP2_PY3 motifs because of the presence of destabilizing residues at various positions. Thus, while both WBP2_PY2 and WBP2_PY3 contain proline at positions -1 and -2 , respectively, WBP2_PY1 contains cysteine at position -1 , WBP1_PY2 contains threonine at position -2 , and WBP1_PY1 contains lysine and phenylalanine at positions -1 and -2 , respectively. Furthermore, while WBP1_PY2, WBP2_PY2, and WBP2_PY3 contain proline at position 2, WBP2_PY1 and WBP1_PY1 contain glycine and alanine, 

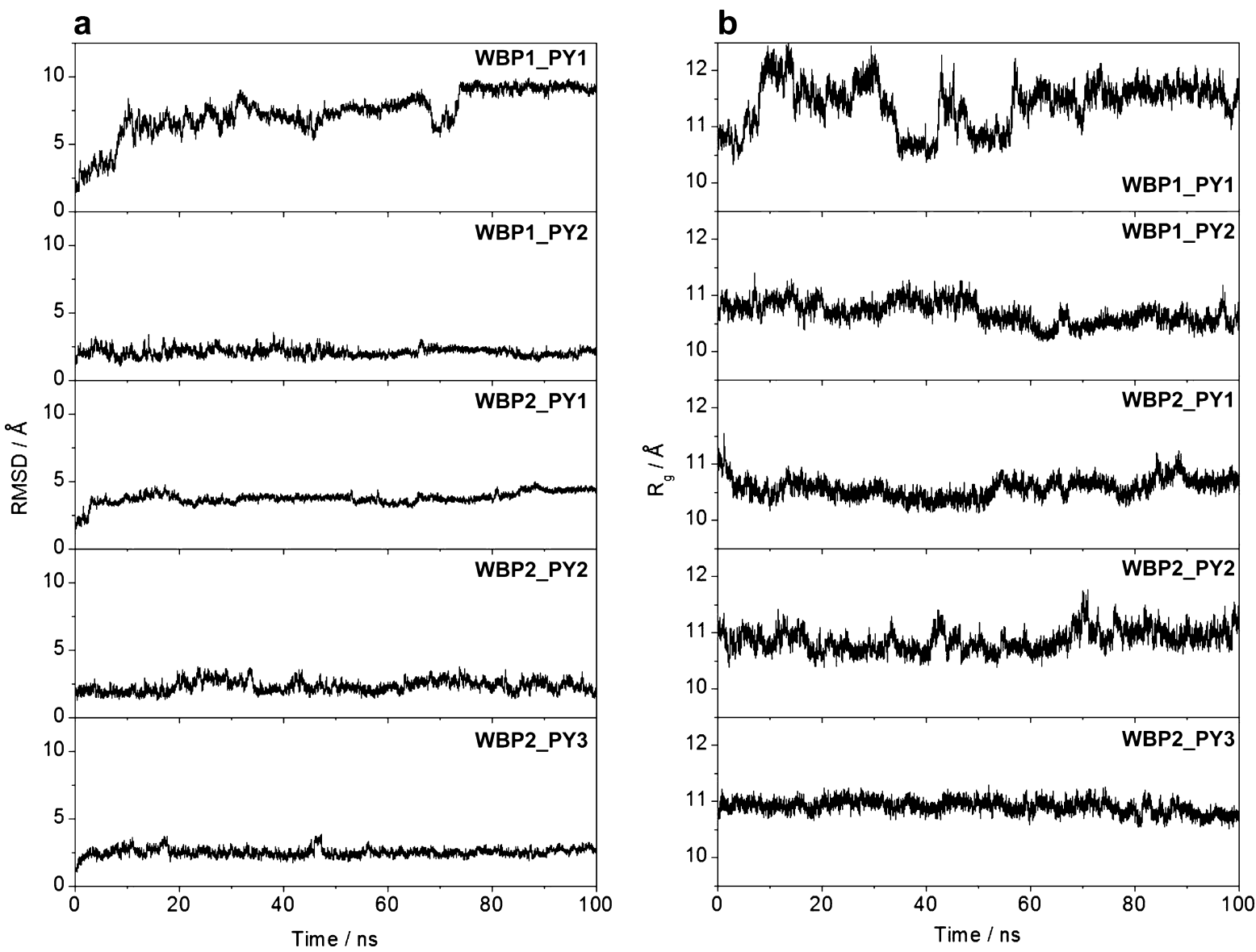

Figure 6. Global behavior of the WW1 domain of YAP2 in complex with various PPXY peptides derived from WBP1 and WBP2 across the corresponding MD trajectory. (a) Root-mean-square deviation (rmsd) of backbone atoms ( $\mathrm{N}, \mathrm{C} \alpha$, and $\mathrm{C}$ ) within each simulated structure relative to the initial modeled structure of the WW1 domain of YAP2 in complex with various peptides as a function of simulation time. (b) Dependence of the radius of gyration $\left(R_{\mathrm{g}}\right)$ of the WW1 domain of YAP2 in complex with various peptides as a function of simulation time.

respectively, at this position. Position 4 also appears to be poorly conserved among various PPX motifs, and the nature of residues at this position could also play a key role in determining the outcome of WW-PPXY interaction. Notably, while WBP2_PY1 and WBP2_PY2 contain proline at position 4 , it is substituted with glutamate in WBP1 PY1, threonine in WBP1 PY2, and tyrosine in WBP2 PY3.

PPXY Peptides Derived from WBP1 and WBP2 Display Conformational Heterogeneity. Our thermodynamic data presented above suggest strongly that nonconsensus residues within and flanking the PPXY peptides are not critical for highaffinity binding to WW domains but may be important for stabilizing the peptide conformation. To further investigate the effect of various residues within and flanking the PPXY motifs on their conformation, we measured and compared secondary structural features of various wild-type and mutant PPXY peptides derived from WBP1 and WBP2 using far-UV CD spectroscopy (Figure 4). Our data suggest that while all peptides show spectral features with minima centered around $195 \mathrm{~nm}$, characteristic of polyproline type II (PPII) helical conformation, ${ }^{36,37}$ there are also discernible differences in the spectral intensities and minima observed in the $200-210 \mathrm{~nm}$ region. This implies that although all peptides overall conform to the PPII helical conformation, they are likley to be conformationally heterogeneous, and such conformational heterogeneity may also account for their binding differences toward the WW domains of YAP2.
Importantly, our $\mathrm{CD}$ data also indicate that alanine substitution of residues within the WBP2 PY3 peptide results in subtle conformational changes, supporting the notion that residues within and flanking the PPXY motifs may play a key role in stabilizing their conformation. Specifically, a more rigid conformation of PPXY peptides, which is likely to be afforded by proline and other residues with bulky side chain moieties, ${ }^{38}$ would result in a lower entropic penalty upon binding to the WW domains than a more flexible conformation resulting from residues such as glycine and alanine with nonbulky side chains. Such a reduction in the entropic penalty would contribute favorably to the overall free energy of WW--PPXY interaction and thereby is likely to result in tighter binding as observed with WBP2_PY2 and WBP2_PY3 peptides. On the other hand, the presence of residues such as glycine and alanine with nonbulky side chains at specific positions within and flanking the PPXY motifs may also be advantageous for allowing the PPXY peptides to attain a close molecular fit within the hydrophobic grooves of the WW domains. In summary, our data suggest that the PPXY motifs that display an intricate balance between rigidity and flexibility are likely to bind to the WW domains with optimal affinity. In this regard, WBP2_PY2 and WBP2_PY3 appear to be optimally suited for binding to the WW domains of YAP2, and such virtue may be attributable to their distinct conformations. This is further supported by the fact that the CD features of WBP2 PY2 and WBP2 PY3 peptides are indeed somewhat distinct in that their spectral 

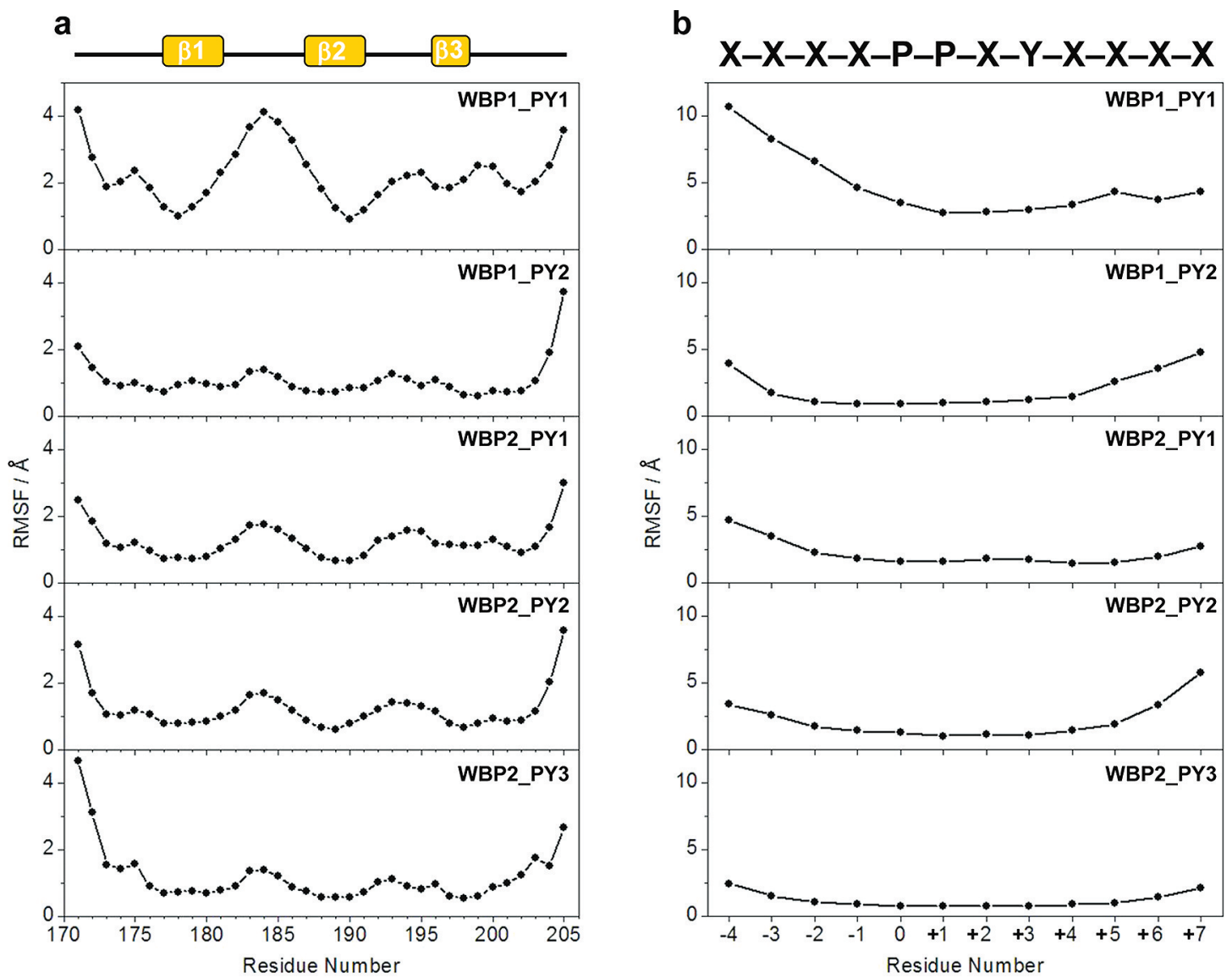

Figure 7. Root-mean-square fluctuations ( $\mathrm{rmsfs}$ ) of backbone atoms $(\mathrm{N}, \mathrm{C} \alpha$, and $\mathrm{C}$ ) averaged over the entire course of the corresponding $\mathrm{MD}$ trajectory for the WW1 domain of YAP2 in complex with various PPXY peptides derived from WBP1 and WBP2. (a) rmsfs within backbone atoms of the WW1 domain in complex with various peptides as a function of WW1 residue number. Strands $\beta 1-\beta 3$ within the WW1 domain are overlaid for reference. (b) rmsfs within backbone atoms of the various peptides in complex with the WW1 domain as a function of peptide residue number. The PPXY motif and the flanking residues are overlaid for reference.

bands in the $200-210 \mathrm{~nm}$ region appear to be much more pronounced compared to those of other peptides.

Structural Models Provide a Physical Basis for the Differential Binding of WW Domains of YAP2 to PPXY Motifs within WBP1 and WBP2. To understand the physical basis of the differential binding of WW domains of YAP2 to various PPXY motifs within WBP1 and WBP2, we modeled 3D structures of WW1 and WW2 domains in complex with the WBP2 PY2 peptide (Figure 5). Our models show that the PPXY peptide adopts the PPII helical conformation and binds within the hydrophobic groove of the antiparallel triplestranded $\beta$-sheet fold of the WW domains in a canonical manner. ${ }^{39-42}$ In agreement with our thermodynamic data presented above, only the consensus residues within the PPXY motif appear to be engaged in key intermolecular contacts with specific residues lining the hydrophobic groove of the WW domains, while nonconsensus residues within and flanking the PPXY motif make no discernible contacts with any residues within the WW domains. Notably, the pyrrolidine moiety of P0, the first proline within the PPXY motif according to the nomenclature presented in panels $\mathrm{b}$ and $\mathrm{c}$ of Figure 1, stacks against the indole side chain of W199 in WW1 and W258 in WW2. The side chains of Y188 and T197 in WW1 and Y247 and T256 in WW2 sandwich the pyrrolidine moiety of P1 within the PPXY motif. The phenyl moiety of Y3, the terminal tyrosine within the PPXY motif, buries deep into the hydrophobic groove and is escorted by side chains of L190, H192, and Q195 in the WW1 domain and I249, H251, and K254 in the WW2 domain. The various interactions between specific side chains in WW domains and the peptide appear to be stabilized by an extensive network of van der Waals contacts and hydrogen bonding. In particular, the $\mathrm{H} \eta$ phenolic hydrogen of $\mathrm{Y} 3$ appears to hydrogen bond with the imidazole $\mathrm{N} \delta 1$ atom of $\mathrm{H} 192$ in the WW1 domain and $\mathrm{H} 251$ in the WW2 domain. Importantly, the lack of a bulky side chain on G5 within the PPXYXG sequence of the WBP2_PY2 peptide not only facilitates close intermolecular contacts between $\mathrm{Y} 3$ and the corresponding triplets within each WW domain but also may play a key role in peptide dynamics necessary for high-affinity binding not observed with peptides lacking a glycine at this position.

Taken together, our structural models suggest that an amino acid residue with a bulky side chain at position 5 may be destabilizing in the context of WW-PPXY interactions. Although nonconsensus residues within and flanking the PPXY motif make no discernible contacts with any residues within the WW domains, some residues at these positions may be more destabilizing than others either through their engagement in unfavorable contacts or by simply compromising the peptide conformation that best fits the hydrophobic groove within the WW domains. It should be noted that W199 and W258, Y188 and Y247, L190 and I249, and H192 and 

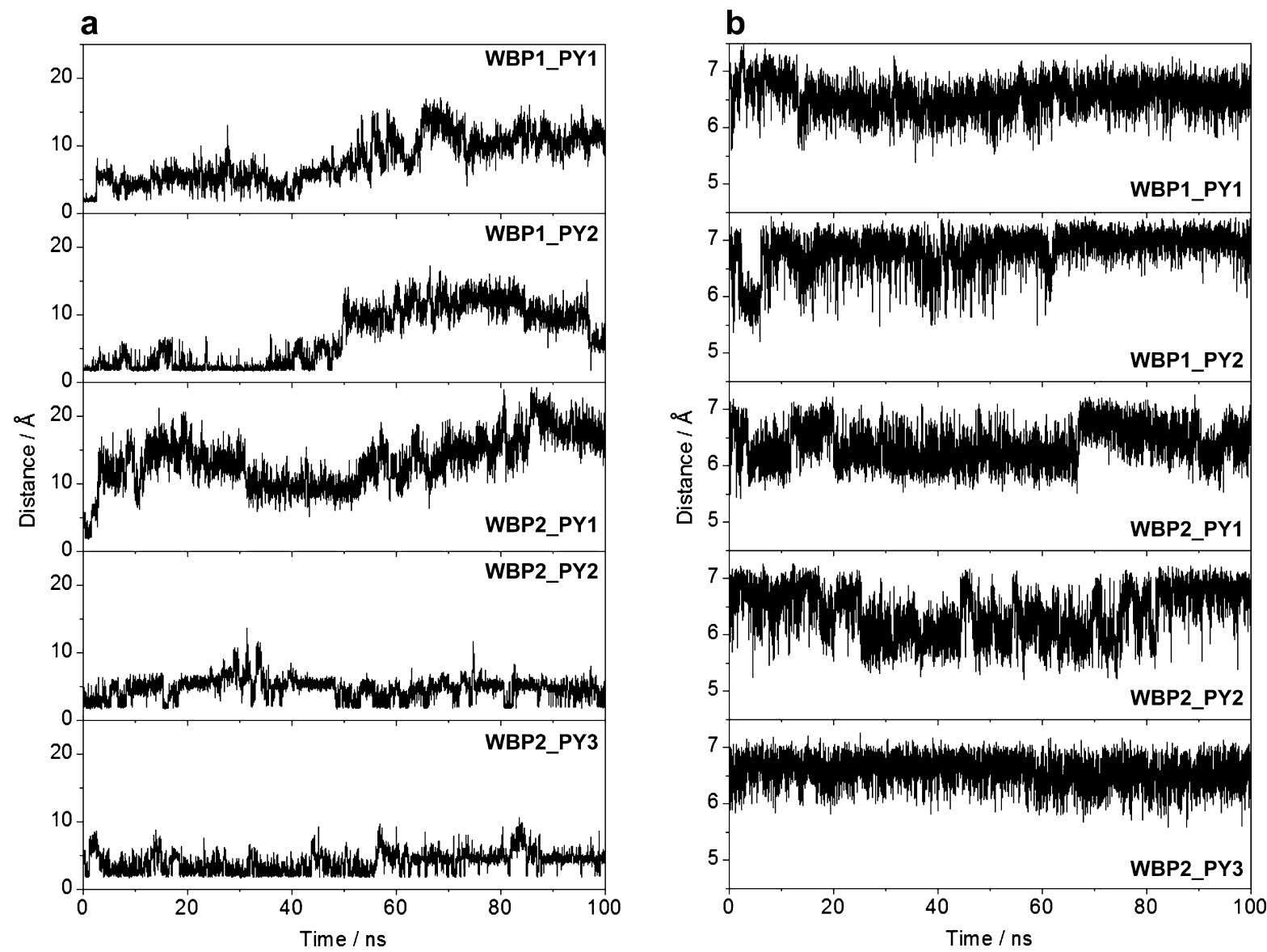

Figure 8. Dependence of local distances between specific atoms of the WW1 domain of YAP2 in complex with various PPXY peptides derived from WBP1 and WBP2 across the corresponding MD trajectory. (a) Distance between the imidazole N $\delta 1$ atom of H192 within the WW1 domain of YAP2 and the phenolic $\mathrm{H} \eta$ of the consensus tyrosine within the PPXY motif of various peptides as a function of simulation time. (b) Distance between the backbone $\mathrm{C} \alpha$ of the consensus tyrosine within the PPXY motif (Y3) and the backbone C $\alpha$ of the residue at position 5 (D5 in WBP1_PY1, V5 in WBP1_PY2, Y5 in WBP2_PY1, G5 in WBP2_PY2, and P5 in WBP2_PY3) within various peptides as a function of simulation time.

H251 lining the hydrophobic grooves within WW domains of YAP2 are highly conserved across the family of WW domains that recognize PPXY ligands. ${ }^{39-42}$

MD Simulations Provide Insights into the Stability and Conformational Dynamics of WW Domains of YAP2 in Complex with PPXY Peptides Derived from WBP1 and WBP2. Protein dynamics play a key role in their biological function. In an effort to understand the role of such motions pertinent to the formation of various complexes between the WW domains of YAP2 and PPXY motifs within WBP1 and WBP2, we conducted MD simulations over tens of nanoseconds, the time regime over which macromolecular motions such as conformational fluctuations and intermolecular movements relevant to their biological function occur. As shown in Figure 6a, the MD trajectories reveal that all but the WBP1_PY1 peptide in complex with the WW1 domain reach structural equilibrium after $\sim 20$ ns with a root-mean-square deviation (rmsd) of $\sim 3 \AA$. The low stability of the WBP1_PY1 peptide in complex with the WW1 domain is further supported by significant fluctuations observed in its radius of gyration $\left(R_{\mathrm{g}}\right)$ relative to those observed for the complexes formed with the other four peptides (Figure $6 b$ ).

An alternative means of assessing the mobility and stability of macromolecular complexes is assessment of the root-meansquare fluctuation (rmsf) of specific atoms over the course of
MD simulation. Panels $\mathrm{a}$ and $\mathrm{b}$ of Figure 7 provide such analysis for the backbone atoms of each residue within both the WW1 domain and each of the five peptides in complex with each other. As expected, the rmsf analysis shows that residues encompassing the $\mathrm{N}$ - and $\mathrm{C}$-termini of both the WW1 domain and each peptide are conformationally flexible relative to residues located within their core regions. Notably, the $\beta 1-\beta 2$ loop that forms one face of the hydrophobic groove within the WW1 domain also appears to be highly flexible, and such internal mobility may be an important facet of the WW domain's ability to mold around a diverse array of PPXY ligands. These observations are consistent with previous dynamics studies conducted on WW domains of YAP. ${ }^{40,43}$ Furthermore, residues within the complex formed between the WBP1_PY1 peptide and WW1 domain overall exude much higher mobility than residues within complexes formed with the other four peptides, implying that the binding of WBP1 PY1 to the WW1 domain results in the formation of a relatively unstable complex in agreement with the MD trajectories shown in Figure 6.

In light of the observation that the $\mathrm{H} \eta$ phenolic hydrogen of Y3 within the PPXY motif of the WBP2_PY2 peptide hydrogen bonds with the imidazole $\mathrm{N} \delta 1$ atom of H192 in the WW1 domain and $\mathrm{H} 251$ in the WW2 domain according to our structural models (Figure 5), we also monitored the stability of 
this hydrogen bond over the course of our MD simulations (Figure 8a). Strikingly, our analysis reveals that this hydrogen bond is stable only for the formation of complexes between the WW1 domain and WBP2_PY2 and WBP2_PY3, the two peptides that bind with much higher affinity than other peptides (Tables 1 and 2). As noted earlier, the fact that WBP2 PY2 binds to the WW domains of YAP2 with the highest affinity partly resides in the ability of $\mathrm{Y} 3$ within this peptide to be buried deeply into the hydrophobic groove of the WW domain because of the presence of a nonbulky and flexible glycine at position 5. This notion is further corroborated by the fact that the backbone region spanning the residues from position 3 to position 5 is indeed more flexible in the WBP2_PY2 peptide than in other peptides (Figure 8b). Accordingly, the flexibility of this backbone region in the WBP2_PY2 peptide may in part account for its tighter binding relative to that of other peptides by allowing it attain a close molecular fit as well as by offsetting the entropic penalty through favorable conformational dynamics. Taken together, our MD simulations corroborate our thermodynamic and structural data in that the complexes formed between the WW1 domain and the WBP2_PY2 and WBP2_PY3 peptides appear to be structurally more stable than other peptides. MD simulations for complexes formed between the WW2 domain of YAP2 and various PPXY peptides derived from WBP1 and WBP2 yielded similar conclusions.

\section{CONCLUSIONS}

YAP integrates a plethora of extracellular signals that converge in the cytosol and routes them to downstream transcription factors and, in so doing, mediates their transcriptional activity in a diverse array of cellular processes in health and disease. ${ }^{3-8}$ Despite such urgency, the molecular mechanism by which YAP recognizes its cellular partners remains largely elusive. Herein, our biophysical analysis provides new insights into YAP-WBP interaction at the atomic level.

Our study shows that both WW domains of YAP2 recognize various PPXY motifs within WBP1 and WBP2 adaptors in a structurally and thermodynamically indistinct manner. This is not surprising given that both WW domains have sequences that are $\sim 50 \%$ identical, and in particular, the residues within the hydrophobic groove involved in side chain interactions with those from the PPXY motifs are virtually identical. It is noteworthy that the WW domains of dystrophin and Nedd4 have a strict requirement of nonconsensus residues flanking the PPXY ligands for high-affinity binding and specificity. ${ }^{41,42}$ The fact that nonconsensus residues within and flanking the PPXY motifs within WBP proteins are not required for high-affinity binding of WW domains of YAP2 appears to be a unique feature of this WW-ligand interaction. It may well be that the WW domains of YAP2 have evolved to be extremely promiscuous and recognize a large repertoire of yet unidentified PPXY ligands. Importantly, a motif search using Prosite at the Expasy server suggests that of all members of the PPXYcontaining family of proteins involved in the Hippo pathway, ${ }^{44}$ only RUNX2 contains the PPXYXG motif, which we have identified here as the most optimal motif in WBP2 for binding to WW domains of YAP2. Interestingly, AMOT and p73, which are also involved in the Hippo pathway, contain the related PPXYXA motif that can also bind with high affinity to WW domains of YAP2 (Tables 3 and 4). On the basis of these considerations, we predict that in addition to WBP2, other
Hippo pathway proteins that may bind YAP with high affinity include RUNX2, AMOT, and p73.

We also point out that the phosphorylation of the signature tyrosine within the PPXY motif has been shown to negatively regulate WW-ligand interactions. ${ }^{9,13}$ Although it is not known if the signature tyrosine within the PPXY motifs of WBP2 is also subject to phosphorylation, Y192 and Y231 within WBP2 have been shown to be phosphorylated in vivo. ${ }^{45}$ In light of this argument, we believe that phosphorylation of the signature tyrosine within the PPXY motifs of WBP proteins may serve as a molecular switch for the regulation of YAP-WBP interaction pertinent to its role in the Hippo pathway. Additionally, Hippo pathway PPXY-containing MST and LATS serine/threonine kinases have also been shown to negatively regulate YAP transcriptional activity. ${ }^{6,22,46}$ It is thus clear that WBP proteins likely compete with MST and LATS for binding to YAP, and their relative intracellular ratios are likely to determine whether YAP is activated or deactivated in response to various stimuli. Although WBP2 contains the PPXYXG motif for optimal binding to WW domains of YAP2 while MST and LATS do not, only a detailed analysis of full-length proteins can reveal whether WBP-YAP interaction is stronger or weaker than MST-YAP or LATS-YAP interactions due to other factors such as bivalent interactions and the formation of multimeric complexes.

Although our analysis of WW domains of YAP2 has been conducted in vitro with short peptides, our data are in agreement with those of previously published studies in which WBP1 and WBP2 were identified as putative YAP-binding partners. ${ }^{12,13}$ Our cell-based data and in vitro pull-down assays also suggest that full-length WBP1 and WBP2 bind to YAP in a specific manner (L. Buffa and Z. Nawaz, unpublished observations). Additionally, the YAP-WBP interaction has also been demonstrated in Drosophila and shown to play a key role in the Hippo pathway. ${ }^{15-17}$ It is noteworthy that the relatively low affinities in the tens to hundreds of micromolar observed for the binding of WW domains of YAP2 to PPXY motifs are characteristic of many WW-ligand interactions in general. ${ }^{19,35,43,47}$ Importantly, these low-affinity interactions may underlie the ability of YAP to bind to WBP proteins as well as other cellular partners in a temporal and reversible manner, a scenario that is the hallmark of signaling cascades and regulatory networks that drive the cellular machinery. Given that we have relied here on short peptides to mimic PPXY motifs in WBP1 and WBP2, caution is warranted in that these motifs may depart from their physiological behavior when treated as short peptides because of the loss of local conformational constraints to which they may be subjected in the context of full-length proteins. Nonetheless, the fact that both WBP1 and WBP2 contain multiple PPXY sites for the binding of WW domains of YAP2 raises the possibility of the formation of YAP2-WBP signaling complexes via a bidentate mechanism, that is, both WW domains of YAP2 binding in a cooperative manner to two individual PPXY sites within WBP1 and WBP2. Such a scenario would clearly enhance the binding affinity of these partners because of the entropic advantage, thereby allowing them to associate with each other at much lower cellular concentrations, perhaps in the submicromolar range in lieu of more than tens of micromolar suggested by our measurements reported here. Accordingly, YAP2-WBP interaction may not only respond in a highly sensitive manner but also be subject to fine-tuning in response to specific extracellular stimuli. 
In conclusion, we have provided here a biophysical framework for understanding a key WW-ligand interaction in the context of cellular signaling circuitry pertinent to health and disease. Our future efforts will focus on unraveling the mechanism of binding of the tandem WW domains of YAP2 to multivalent PPXY ligands derived from WBP proteins.

\section{AUTHOR INFORMATION}

\section{Corresponding Author}

*E-mail: amjad@farooqlab.net. Telephone: (305) 243-2429. Fax: (305) 243-3955.

\section{Funding}

This work was supported by National Institutes of Health Grants R01-GM083897 (to A.F.) and R01-DK079217 (to Z.N.), funds from the USylvester Braman Family Breast Cancer Institute (to A.F. and Z.N.), Pennsylvania Breast Cancer Coalition Grants 60707 (to M.S.) and 9200903 (to M.S.), and funds from the Geisinger Clinic (to M.S.). C.B.M. is a recipient of a postdoctoral fellowship from the National Institutes of Health (T32-CA119929).

\section{ABBREVIATIONS}

CD, circular dichroism; E6AP, E6-associated protein; ITC, isothermal titration calorimetry; LIC, ligation-independent cloning; MD, molecular dynamics; MM, molecular modeling; PPII, polyproline type II; SEC, size-exclusion chromatography; WBP1, WW-binding protein 1; WBP2, WW-binding protein 2; YAP2, YES-associated protein 2.

\section{REFERENCES}

(1) Sudol, M. (1994) Yes-associated protein (YAP65) is a prolinerich phosphoprotein that binds to the $\mathrm{SH} 3$ domain of the Yes protooncogene product. Oncogene 9, 2145-2152.

(2) Sudol, M., Bork, P., Einbond, A., Kastury, K., Druck, T., Negrini, M., Huebner, K., and Lehman, D. (1995) Characterization of the mammalian YAP (Yes-associated protein) gene and its role in defining a novel protein module, the WW domain. J. Biol. Chem. 270, 1473314741.

(3) Yagi, R., Chen, L. F., Shigesada, K., Murakami, Y., and Ito, Y. (1999) A WW domain-containing yes-associated protein (YAP) is a novel transcriptional co-activator. EMBO J. 18, 2551-2562.

(4) Komuro, A., Nagai, M., Navin, N. E., and Sudol, M. (2003) WW domain-containing protein YAP associates with ErbB-4 and acts as a co-transcriptional activator for the carboxyl-terminal fragment of ErbB4 that translocates to the nucleus. J. Biol. Chem. 278, 33334-33341.

(5) Zhao, B., Ye, X., Yu, J., Li, L., Li, W., Li, S., Lin, J. D., Wang, C. Y., Chinnaiyan, A. M., Lai, Z. C., and Guan, K. L. (2008) TEAD mediates YAP-dependent gene induction and growth control. Genes Dev. 22, 1962-1971.

(6) Hao, Y., Chun, A., Cheung, K., Rashidi, B., and Yang, X. (2008) Tumor suppressor LATS1 is a negative regulator of oncogene YAP. J. Biol. Chem. 283, 5496-5509.

(7) Zhao, B., Wei, X., Li, W., Udan, R. S., Yang, Q., Kim, J., Xie, J., Ikenoue, T., Yu, J., Li, L., Zheng, P., Ye, K., Chinnaiyan, A., Halder, G., Lai, Z. C., and Guan, K. L. (2007) Inactivation of YAP oncoprotein by the Hippo pathway is involved in cell contact inhibition and tissue growth control. Genes Dev. 21, 2747-2761.

(8) Bertini, E., Oka, T., Sudol, M., Strano, S., and Blandino, G. (2009) YAP: At the crossroad between transformation and tumor suppression. Cell Cycle 8, 49-57.

(9) Sudol, M. (2010) Newcomers to the WW Domain-Mediated Network of the Hippo Tumor Suppressor Pathway. Genes Cancer 1, 1115-1118.

(10) Sudol, M., and Harvey, K. F. (2010) Modularity in the Hippo signaling pathway. Trends Biochem. Sci. 35, 627-633.
(11) Morin-Kensicki, E. M., Boone, B. N., Howell, M., Stonebraker, J. R., Teed, J., Alb, J. G., Magnuson, T. R., O'Neal, W., and Milgram, S. L. (2006) Defects in yolk sac vasculogenesis, chorioallantoic fusion, and embryonic axis elongation in mice with targeted disruption of Yap65. Mol. Cell. Biol. 26, 77-87.

(12) Chen, H. I., and Sudol, M. (1995) The WW domain of Yesassociated protein binds a proline-rich ligand that differs from the consensus established for Src homology 3-binding modules. Proc. Natl. Acad. Sci. U.S.A. 92, 7819-7823.

(13) Chen, H. I., Einbond, A., Kwak, S. J., Linn, H., Koepf, E., Peterson, S., Kelly, J. W., and Sudol, M. (1997) Characterization of the WW domain of human yes-associated protein and its polyprolinecontaining ligands. J. Biol. Chem. 272, 17070-17077.

(14) Dhananjayan, S. C., Ramamoorthy, S., Khan, O. Y., Ismail, A., Sun, J., Slingerland, J., O'Malley, B. W., and Nawaz, Z. (2006) WW domain binding protein-2, an E6-associated protein interacting protein, acts as a coactivator of estrogen and progesterone receptors. Mol. Endocrinol. 20, 2343-2354.

(15) Zhang, X., Milton, C. C., Humbert, P. O., and Harvey, K. F. (2009) Transcriptional output of the Salvador/warts/hippo pathway is controlled in distinct fashions in Drosophila melanogaster and mammalian cell lines. Cancer Res. 69, 6033-6041.

(16) Zhang, X., Milton, C. C., Poon, C. L., Hong, W., and Harvey, K. F. (2011) Wbp2 cooperates with Yorkie to drive tissue growth downstream of the Salvador-Warts-Hippo pathway. Cell Death Differ. 18, 1346-1355.

(17) Grusche, F. A., Degoutin, J. L., Richardson, H. E., and Harvey, K. F. (2011) The Salvador/Warts/Hippo pathway controls regenerative tissue growth in Drosophila melanogaster. Dev. Biol. 350, 255-266.

(18) Sudol, M., Chen, H., Bougeret, C., Einbond, A., and Bork, P. (1995) Characterization of a novel protein-binding module: The WW domain. FEBS Lett. 369, 67-71.

(19) Kay, B. K., Williamson, M. P., and Sudol, M. (2000) The importance of being proline: The interaction of proline-rich motifs in signaling proteins with their cognate domains. FASEB J. 14, 231-241.

(20) Sudol, M., and Hunter, T. (2000) NeW wrinkles for an old domain. Cell 103, 1001-1004.

(21) Sudol, M. (2004) WW domain. In Modular Protein Domains (Cesareni, G., Gimona, M., Sudol, M., and Yaffe, M., Eds.) pp 59-72, Wiley VCH, Verlag GmbH \& Co., Berlin.

(22) Oka, T., Mazack, V., and Sudol, M. (2008) Mst2 and Lats kinases regulate apoptotic function of Yes kinase-associated protein (YAP). J. Biol. Chem. 283, 27534-27546.

(23) Gasteiger, E., Hoogland, C., Gattiker, A., Duvaud, S., Wilkins, M. R., Appel, R. D., and Bairoch, A. (2005) Protein Identification and Analysis Tools on the ExPASy Server. In The Proteomics Protocols Handbook (Walker, J. M., Ed.) pp 571-607, Humana Press, Totowa, NJ.

(24) Wiseman, T., Williston, S., Brandts, J. F., and Lin, L. N. (1989) Rapid measurement of binding constants and heats of binding using a new titration calorimeter. Anal. Biochem. 179, 131-137.

(25) Marti-Renom, M. A., Stuart, A. C., Fiser, A., Sanchez, R., Melo, F., and Sali, A. (2000) Comparative Protein Structure Modeling of Genes and Genomes. Annu. Rev. Biophys. Biomol. Struct. 29, 291-325.

(26) Carson, M. (1991) Ribbons 2.0. J. Appl. Crystallogr. 24, 958961.

(27) Van Der Spoel, D., Lindahl, E., Hess, B., Groenhof, G., Mark, A. E., and Berendsen, H. J. (2005) GROMACS: Fast, flexible, and free. J. Comput. Chem. 26, 1701-1718.

(28) Hess, B. (2008) GROMACS 4: Algorithms for Highly Efficient, Load-Balanced, and Scalable Molecular Simulation. J. Chem. Theory Comput. 4, 435-447.

(29) Jorgensen, W. L., and Tirado-Rives, J. (1988) The OPLS Force Field for Proteins: Energy Minimizations for Crystals of Cyclic Peptides and Crambin. J. Am. Chem. Soc. 110, 1657-1666.

(30) Kaminski, G. A., Friesner, R. A., Tirado-Rives, J., and Jorgensen, W. L. (2001) Evaluation and Reparametrization of the OPLS-AA Force Field for Proteins via Comparison with Accurate Quantum Chemical Calculations on Peptides. J. Phys. Chem. B 105, 6474-6487. 
(31) Toukan, K., and Rahman, A. (1985) Molecular-dynamics study of atomic motions in water. Phys. Rev. B 31, 2643-2648.

(32) Berendsen, H. J. C., Grigera, J. R, and Straatsma, T. P. (1987) The Missing Term in Effective Pair Potentials. J. Phys. Chem. 91, 6269-6271.

(33) Darden, T. A., York, D., and Pedersen, L. (1993) Particle mesh Ewald: An N.log(N) method for Ewald sums in large systems. J. Chem. Phys. 98, 10089-10092.

(34) Hess, B., Bekker, H., Berendsen, H. J. C., and Fraaije, J. G. E. M. (1997) LINCS: A linear constraint solver for molecular simulations. J. Comput. Chem. 18, 1463-1472.

(35) Linn, H., Ermekova, K. S., Rentschler, S., Sparks, A. B., Kay, B. K., and Sudol, M. (1997) Using molecular repertoires to identify highaffinity peptide ligands of the WW domain of human and mouse YAP. Biol. Chem. 378, 531-537.

(36) Rabanal, F., Ludevid, M. D., Pons, M., and Giralt, E. (1993) CD of proline-rich polypeptides: Application to the study of the repetitive domain of maize glutelin-2. Biopolymers 33, 1019-1028.

(37) Kelly, S. M., Jess, T. J., and Price, N. C. (2005) How to study proteins by circular dichroism. Biochim. Biophys. Acta 1751, 119-139.

(38) MacArthur, M. W., and Thornton, J. M. (1991) Influence of proline residues on protein conformation. J. Mol. Biol. 218, 397-412.

(39) Macias, M. J., Hyvonen, M., Baraldi, E., Schultz, J., Sudol, M., Saraste, M., and Oschkinat, H. (1996) Structure of the WW domain of a kinase-associated protein complexed with a proline-rich peptide. Nature 382, 646-649.

(40) Pires, J. R., Taha-Nejad, F., Toepert, F., Ast, T., Hoffmuller, U., Schneider-Mergener, J., Kuhne, R., Macias, M. J., and Oschkinat, H. (2001) Solution structures of the YAP65 WW domain and the variant L30 $\mathrm{K}$ in complex with the peptides GTPPPPYTVG, N-(n-octyl)GPPPY and PLPPY and the application of peptide libraries reveal a minimal binding epitope. J. Mol. Biol. 314, 1147-1156.

(41) Huang, X., Poy, F., Zhang, R., Joachimiak, A., Sudol, M., and Eck, M. J. (2000) Structure of a WW domain containing fragment of dystrophin in complex with $\beta$-dystroglycan. Nat. Struct. Biol. 7, 634638.

(42) Kanelis, V., Rotin, D., and Forman-Kay, J. D. (2001) Solution structure of a Nedd4 WW domain-ENaC peptide complex. Nat. Struct. Biol. 8, 407-412.

(43) Webb, C., Upadhyay, A., Giuntini, F., Eggleston, I., FurutaniSeiki, M., Ishima, R., and Bagby, S. (2011) Structural features and ligand binding properties of tandem WW domains from YAP and TAZ, nuclear effectors of the Hippo pathway. Biochemistry 50, 33003309.

(44) Salah, Z., and Aqeilan, R. I. (2011) WW domain interactions regulate the Hippo tumor suppressor pathway. Cell Death Dis. 2, e172.

(45) Lim, S. K., Orhant-Prioux, M., Toy, W., Tan, K. Y., and Lim, Y. P. (2011) Tyrosine phosphorylation of transcriptional coactivator WW-domain binding protein 2 regulates estrogen receptor $\alpha$ function in breast cancer via the Wnt pathway. FASEB J. 25, 3004-3018.

(46) Zhang, J., Smolen, G. A., and Haber, D. A. (2008) Negative regulation of YAP by LATS1 underscores evolutionary conservation of the Drosophila Hippo pathway. Cancer Res. 68, 2789-2794.

(47) Macias, M. J., Wiesner, S., and Sudol, M. (2002) WW and SH3 domains, two different scaffolds to recognize proline-rich ligands. FEBS Lett. 513, 30-37. 